\title{
Evidential reasoning based decision system to select health care location
}

\author{
Md. Mahashin Mia, Atiqur Rahman, Mohammad Shahadat Hossain
}

Department of Computer Science and Engineering, University of Chittagong, Bangladesh

\begin{abstract}
Article Info
Article history:

Received Mar 20, 2020

Revised Apr 24, 2020

Accepted May 11, 2020

\section{Keywords:}

Belief rule base

Evidential reasoning

Intelligent decision system

Multiple criteria decision

analysis

Uncertainty

ABSTRACT

The general public's demand of Bangladesh for safe health is rising promptly with the improvement of the living standard. However, the allocation of limited and unbalanced medical resources is deteriorating the assurance of safe health of the people. Therefore, the new hospital construction with rational allocation of resources is imminent and significant. The site selection for establishing a hospital is one of the crucial policy-related decisions taken by planners and policy makers. The process of hospital site selection is inherently complicated because of this involves many factors to be measured and evaluated. These factors are expressed both in objective and subjective ways where as a hierarchical relationship exists among the factors. In addition, it is difficult to measure qualitative factors in a quantitative way, resulting incompleteness in data and hence, uncertainty. Besides it is essential to address the subject of uncertainty by using apt methodology; otherwise, the decision to choose a suitable site will become inapt. Therefore, this paper demonstrates the application of a novel method named belief rulebased inference methodology-RIMER base intelligent decision system(IDS), which is capable of addressing suitable site for hospital by taking account of large number of criteria, where there exist factors of both subjective and objective nature.
\end{abstract}

This is an open access article under the CC BY-SA license.

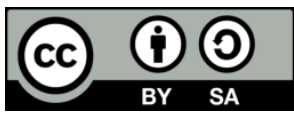

\section{Corresponding Author:}

Atiqur Rahman,

Department of Computer Science \& Engineering,

University of Chittagong,

Chittagong-4331, Bangladesh.

Email: bulbul.cse.cu@gmail.com

\section{INTRODUCTION}

When we attempt to select suitable site for hospital, it involves multiple criterions such as, location, safety, environment, parking space, Land cost, Risk, transportation cost and utility cost etc. which are quantitative and qualitative in nature $[1,2]$. Numerical data which uses numbers is considered as quantitative data and can be measured with $100 \%$ certainty [2]. On the contrary, qualitative data is descriptive in nature, which defines some concepts or imprecise characteristics or quality of things [3]. Hence, this data can't describe a thing with certainty since it lacks the precision and inherits ambiguity, ignorance, vagueness. Consequently, it can be argued that qualitative data involves uncertainty since it is difficult to measure concepts or characteristics or quality of a thing with $100 \%$ certainty. "Quality of Location" is an example of equivocal term since it is an example of linguistic term. Hence, it is difficult to extract its correct semantics (meaning). However, this can be evaluated using some referential value such as excellent, good, average and bad. Therefore, it can be seen that qualitative criterions which have been considered in selecting hospital location involves lot of uncertainties and they should be treated with appropriate methodology is 
RIMER, which is connect to Evidential reasoning (ER) is a multi-criteria decision analysis (MCDA) method [4]. ER deals with problems, consisting of both quantitative and qualitative criteria under various uncertainties such as incomplete information, vagueness, ambiguity [4]. The ER approach, developed based on decision theory in particular utility theory [5], artificial intelligence in particular the theory of evidence [5,6]. It uses a belief structure to model a judgment with uncertainty. Qualitative attribute such as location or safety needs to be evaluated using some linguistic referential value such as excellent, average, good and bad etc [5,6]. This requires human judgment for evaluating the attributes based on the mentioned referential value. In this way, the issue of uncertainty can be addressed and more accurate and robust decision can be made. The belief rule-based inference methodology-RIMER [7] has addressed such issue by proposing a belief structure, which assigns degree of belief in the various referential values of the attributes. Road Map: In section 2 will briefly represent belief rule base inference methodology-RIMER. Section 3 will demonstrate the application of BRB in hospital site selection assessment problem. Section 4 will represent the results and achievement. Finally section 5 will conclude the research.

\section{RIMER TO DEVELOP IDS}

In RIMER, Belief Rule Base (BRB) can capture complicated nonlinear causal relationships between antecedent attributes and consequents, which is not possible in traditional IF-THEN rules. BRB is used to model domain specific knowledge under uncertainty, and the ER approach is employed to facilitate inference. This section introduces BRB as a knowledge representation schema under uncertainty as well as inference procedures of RIMER.

\subsection{Modeling doain knowledge using BRB}

Belief Rules are the key constituents of a BRB, which include belief degree. This is the extended form of traditional IF-THEN rules. In a belief rule, each antecedent attribute takes referential values and each possible consequent is associated with belief degrees [8]. The knowledge representation parameters are rule weights, attribute weights and belief degrees in consequent attribute, which are not available in traditional IFTHEN rules. A belief rule can be defined in the following way.

$$
\begin{aligned}
& R_{k}:\left\{\begin{array}{l}
I F\left(P_{1} \text { is } A_{1}^{k}\right) \cap \ldots \cap P_{T_{k}} \text { is } A_{T_{k}}^{k} \\
T H E N\left\{\left(C_{1}, \beta_{1 k}\right),\left(C_{2}, \beta_{2 k}\right), \ldots,\left(C_{N}, \beta_{N k}\right)\right\}
\end{array}, \quad R_{k}:\left(\beta_{j k} \geq 0, \sum_{j=1}^{N} \beta_{j k} \leq 1\right) \text { with a rule } \theta_{k},\right. \text { attribute } \\
& \text { weight } \delta_{k 1}, \delta_{k 2}, \delta_{k 3}, \ldots, \delta_{k T_{k}} k \in\{1, \ldots, L\} \text { weight } \delta_{k 1}, \delta_{k 2}, \delta_{k 3}, \ldots, \delta_{k T_{k}} k \in\{1, \ldots, L\}
\end{aligned}
$$

Where $\boldsymbol{P}_{1}, \boldsymbol{P}_{2}, \boldsymbol{P}_{3} \ldots P_{T k} P_{T k}$ represent the antecedent attributes in the $\boldsymbol{k}$ th rule. represents one of $A_{i}^{k}\left(i=1, \ldots, \mathrm{T}_{k}, k=1, \ldots, \mathrm{L}\right) A_{i}^{k}\left(i=1, \ldots, \mathrm{T}_{k}, k=1, \ldots, \mathrm{L}\right)$ the referential values of the $i$ th antecedent attribute $\boldsymbol{P}_{\boldsymbol{i}}$ in the $k$ th rule. $C_{j} C_{j} C_{j}$ is one of the consequent reference values of the belief rule. $\beta_{j k}(j=1, \ldots, N, k=1, \ldots, L$ $\beta_{j k}\left(j=1, \ldots, N, k=1, \ldots, L \beta_{j k}(j=1, \ldots, N, k=1, \ldots, L\right.$ is one of the the belief degrees to which the consequent reference value $C_{j} C_{j} C_{j}$ is believed to be true. If $1 \sum_{j}^{N}=1 \beta_{1 k}=1 \sum_{j}^{N}=1 \beta_{1 k}=1$ the $k$ th rule is said to be complete; otherwise, it is incomplete. $\boldsymbol{T}_{\boldsymbol{k}}$ is the total number of antecedent attributes used in kth rule $\boldsymbol{L}$ is the number of all belief rules in the rule base. $N$ is the number of all possible consequent in the rule base. For example a belief rule to assess accessibility term for hospital can be written in the following way.

$$
R_{k}:\left\{\begin{array}{l}
\text { IF Transportation cost is good AND traffic_access is good Neutral_Location is good } \\
\text { THEN Accessibility is } \\
\{(\text { Excellent, }(0.00)),(\text { good, }(1.00)),(\text { average },(0.00))\}
\end{array}\right.
$$

Where $\{($ Excellent, 0.00), (Good, 1.00), (Average, 0.00) $\}$ is a belief distribution for accessibility consequent, stating that the degree of belief associated with Excellent is 0\%, 100\% with Good and 0\% with Average. In this belief rule, the total degree of belief is $(0+1+0)=1$, hence that the assessment is complete.

\subsection{BRB Inference using ER}

The ER approach [9] developed to handle multiple attribute decision analysis (MADA) problem having both qualitative and quantitative attributes. Different from traditional MADA approaches, ER presents MADA problem by using a decision matrix, or a belief expression matrix, in which each attribute of an alternative described by a distribution assessment using a belief structure. The inference procedures in $\mathrm{BRB}$ inference system consists of various components such as input transformation, rule activation weight 
calculation, rule update mechanism, followed by the aggregation of the rules of a BRB by using ER [10-13]. The input transformation of a value of an antecedent attribute $\mathrm{P}_{\mathrm{i}}$ consists of distributing the value into belief degrees of different referential values of that antecedent. This is equivalent to transforming an input into a distribution on referential values of an antecedent attribute by using their corresponding belief degrees [14]. The ith value of an antecedent attribute at instant point in time can equivalently be transformed into a distribution over the referential values, defined for the attribute by using their belief degrees.

The input value of $\mathbf{P}_{\mathbf{i}}, \mathbf{P}_{\mathbf{i}}$ which is the ith antecedent attribute of a rule, along with its belief degree $\varepsilon_{i} \varepsilon_{i}$ is shown by (3). The belief degree $\varepsilon_{i} \varepsilon_{i}$ to the input value is assigned by the expert in this research.

$$
H\left(P_{i}, \varepsilon_{i}\right)=\left\{\left(A_{i j}, a_{i j}\right), j=1, \ldots, j_{i}\right\}, i=1, \ldots, T_{k}
$$

Here $\mathrm{H}$ is used to show the assessment of the belief degree assigned to the input value of the antecedent attribute. In (3) $A_{i j} A_{i j}$ (ith value) is the jth referential value of the input $\mathbf{P}_{\mathbf{i}}, \mathbf{P}_{\mathbf{i} .} a_{i j} a_{i j}$ is the belief degree to the referential value $A_{i j} A_{i j}$ with $a_{i j} \geq 0 a_{i j} \geq 0 \sum_{j=1}^{j_{1}} a_{i j} \leq 1\left(i=1, \ldots, T_{k}\right) . \sum_{j=1}^{j_{1}} a_{i j} \leq 1(i=$ $\left.1, \ldots, T_{k}\right)$, and $\boldsymbol{j}_{\boldsymbol{i}} \boldsymbol{j}_{\boldsymbol{i}}$ is the number of the referential values.

For example, the input 0.82 for Accessibility is equivalently transformed to (Excellent, 0.81), (Good, 0.19), (Average, 0.00)\}. The input value of an antecedent attribute is collected from the expert in terms of linguistic values such as 'Excellent', 'Good', 'Average' and 'Bad'. This linguistic value is then assigned degree of belief $\varepsilon_{i} \varepsilon_{i}$ by taking account of expert judgment. This assigned degree of belief is then distributed in terms of belief degree $a_{i j} a_{i j}$ of the different referential values $A_{i j} A_{i j}$ [Excellent, Good, Average, Bad] of the antecedent attribute. The above procedure of input transformation is elaborated by (4) (5) given below. However, when a hospital is located $1.1 \mathrm{~km}$ of the place, it can be both excellent and average. However, it is important for us to know, with what degree of belief it is excellent and with what degree of belief it is average. This phenomenon can be calculated with the following formula.

$$
\begin{aligned}
& \beta_{n, i}=\frac{h_{n+1}-h}{h_{n+1}, i-h_{n, i}}, \beta_{n+1, i}=1-\beta_{n, i} \\
& \text { if } \quad h_{n, i} \leq h \leq h_{n+1, i}
\end{aligned}
$$

Here, the degree of belief $\beta_{n, i}$ is associated with the evaluation grade 'average' while $\beta_{n+1, i}$ is associated with the upper level evaluation grade i.e. excellent. The value of $h_{n+1}$ is the value related to excellent, which is considered as $1 \mathrm{~km}$ i.e. the location of the hospital. The value of $h_{n+1}$ is related to average, which is 1.5 $\mathrm{km}$. Hence, applying (2) the distribution of the degree of belief with respect to $1.3 \mathrm{Km}$ of the location of the hospital can be assessed by using (2) and the result is given:

$\{($ Excellent, 0.4), (Good, 0.6), (Average, 0), (Bad, 0) $\}$,

When the $\boldsymbol{k}$ th rule is activated, the weight of activation of the $\boldsymbol{k}$ th rule, $\boldsymbol{\omega}_{\mathbf{k}}, \boldsymbol{\omega}_{\mathbf{k}}$ is calculated by using the flowing formula [15].

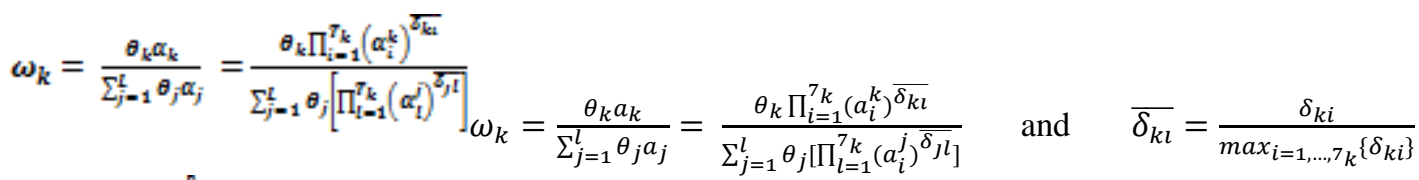

$$
\begin{aligned}
& \overline{\delta_{k L}}=\frac{\delta_{k i}}{\max _{i=1-n} x_{k}\left[\delta_{k i}\right]}
\end{aligned}
$$

Where $\overline{\delta_{k l}} \overline{\delta_{k l}}$ is the relative weight of $\mathrm{P}_{\mathrm{i}} \mathrm{P}_{\mathrm{i}}$ used in the $\boldsymbol{k}$ th rule, which is calculated by dividing weight of $P_{i} P_{i} \overline{\delta_{k l}} \overline{\delta_{k l}}$ with maximum weight of all the antecedent attributes of the kth rule. By doing so, the value of becomes normalize, meaning that the range of its value should be between 0 and $1 . a_{k}=$ $\prod_{i=1}^{T_{k}}\left(a_{i}^{k}\right) \overline{\delta_{k l}} a_{k}=\prod_{i=1}^{T_{k}}\left(a_{i}^{k}\right) \overline{\delta_{k l}}$ is the combined matching degree, which is calculated by using multiplicative aggregation function. When the $k$ th rule as given in (1) is activated, the incompleteness of the consequent of a rule can also result from its antecedents due to lack of data. An incomplete input for an attribute will lead to an incomplete output in each of the rules in which the attribute is used. The original belief degree $\overline{\beta_{1 k}} \overline{\beta_{1 k}}$ in the $\boldsymbol{i}$ th consequent $\mathbf{C}_{\mathbf{i}} \mathbf{C}_{\mathbf{i}}$ of the $\boldsymbol{k}$ th rule is updated based on the actual input information as [10-13]. 


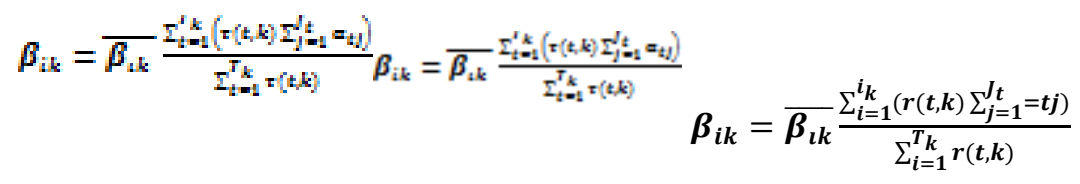

Where, $(t, k)=\left\{\begin{array}{ll}1, & \text { if } p_{i} \text { is used in defining } R_{k}\left(t=1, \ldots, T_{k}\right) \\ 0, & \text { otherwise }\end{array}(t, k)= \begin{cases}1, & \text { if } p_{i} \text { is used in defining } R_{k}\left(t=1, \ldots, T_{k}\right) \\ 0, & \text { otherwise }\end{cases}\right.$ $\overline{\beta_{1 k}} \overline{\beta_{1 k}}$ is the original belief degree and $\boldsymbol{\beta}_{\mathbf{i k}} \boldsymbol{\beta}_{\mathbf{i k}}$ is the updated belief degree.

Due to the incomplete input for 'Accessibility',the belief degree of the connected rules needs to be modified to show the incompleteness by using (7)

$$
\beta_{i k} \equiv \bar{\beta}_{i k} \frac{1.6}{2}=\bar{\beta}_{i k} * 0.8, \quad i=1,2,3 ; k=1, \ldots 9 .
$$

Therefore $0<\sum_{i=1}^{3} \beta_{i k}<1$ for all rules that are associated with 'Cost'. Using the subrule base, the assessment result for 'Accessibility' is obtained using IDS system as Accessibility: $\{($ Excellent, 0.66),(Good,0.23),(Average,0.02),(Bad,0.00), (Unknown,0.09) $\}$

Where, "Unknown" in the above result means that the output is also incomplete input. ER approach is used to aggregate all the packet antecedents of the $\boldsymbol{L}$ rules to obtain the degree of belief of each referential values of the consequent attribute by taking account of given input values $\mathbf{P}_{\mathbf{i}} \mathbf{P}_{\mathbf{i}}$ of antecedent attributes. This aggregation can be carried out either using recursive or analytical approach. In this research analytical approach [14] has been considered since it is computationally efficient than recursive approach [10-12], because analytical approach deal with all parameter such as rule weight, attribute weight ,belief degree, utility etc. For this why there is no chance of absence of any parameter. The conclusion $O(Y)$, consisting of referential values of the consequent attribute, is generated. Equation (9) as given below illustrates the above phenomenon:

$$
O(Y)=S\left(p_{i}\right)=\left\{\left(C_{j}, \beta_{j}\right), j=1, \ldots, N\right\} O(Y)=S\left(p_{i}\right)=\left\{\left(C_{j}, \beta_{j}\right), j=1, \ldots, N\right\}
$$

Where $\boldsymbol{\beta}_{\mathbf{j}} \boldsymbol{\beta}_{\mathbf{j}}$ denotes the belief degree associated with one of the consequent reference values such as $\mathbf{C}_{\mathbf{j}} \mathbf{C}_{\mathbf{j}}$ The $\boldsymbol{\beta}_{\mathbf{j}} \boldsymbol{\beta}_{\mathbf{j}}$ is calculating by analytical format of the ER algorithm [3] as illustrated in (10).

$$
\begin{aligned}
& \beta_{j}=\frac{\mu \times\left[\prod_{k=1}^{L}\left(\left(\omega_{k} \beta_{j k}+1-\omega_{k} \sum_{j=1}^{N} \beta_{j k}\right)\right)-\prod_{k=1}^{L}\left(1-\omega_{k} \sum_{j=1}^{N} \beta_{j k}\right)\right]}{1-\mu \times\left[\prod_{k=1}^{L} 1-\omega_{k}\right]} \text { With } \mu=\left[\sum _ { j = 1 } ^ { N } \prod _ { k = 1 } ^ { L } \left(\omega_{k} \beta_{j k}+1-\right.\right. \\
&\left.\left.\omega_{k} \sum_{j=1}^{N} \beta_{j k}\right)-(N-1) \times \prod_{k=1}^{n}\left(1-\omega_{k} \sum_{j=1}^{N} \beta_{j k}\right)\right]
\end{aligned}
$$

The final combined result or output generated by ER is represented by $\left\{\left(C_{1}, \beta_{1}\right),\left(C_{2}, \beta_{1}\right),\left(C_{3}, \beta_{1}\right), \ldots,\left(C_{N}, \beta_{N}\right)\right\}\left\{\left(C_{1}, \beta_{1}\right),\left(C_{2}, \beta_{1}\right),\left(C_{3}, \beta_{1}\right), \ldots,\left(C_{N}, \beta_{N}\right)\right\}$ Where $\boldsymbol{\beta}_{\mathbf{j}} \boldsymbol{\beta}_{\mathbf{j}}$ is the final belief degree attached to the $j$ th referential value of $\mathbf{C}_{\mathbf{j}} \mathbf{C}_{\mathbf{j}}$ the consequent attribute, obtained after combining all activated rules in the BRB by using ER.

\subsection{Output of the BRB system}

The output of the BRB system is not crisp/numerical value. Hence, this output can be converted into crisp/numerical value by assigning utility score to each referential value of the consequent attribute [16].

$$
H\left(A^{*}\right)=\sum_{j=1}^{N} u\left(C_{j}\right) B_{j} \quad H\left(A^{*}\right)=\sum_{j=1}^{N} u\left(C_{j}\right) B_{j}
$$

Where $H\left(A^{*}\right) H\left(A^{*}\right)$ is the expected score expressed as numerical value and $u\left(C_{j}\right) u\left(C_{j}\right)$ is the utility score of each referential value. For example, in this paper the overall assessment result is $\{($ Excellent,0.55),(Good, 0.25),(Average,0.20),(Bad,0.00)\}for selecting hospital, then the expected utility score is 0.675 or $68 \%$ which represents good risk for suitable hospital location.

In this paper the RIMER methodology to address various type of uncertainty such as incompleteness, ignorance and impreciseness by using (7) and (12). The incompleteness as mentioned occurs due to ignorance, meaning that belief degree has not been assigned to any specific evaluation grade and this can be represented using (12). 


$$
\beta_{H}=1-\sum_{n=1}^{N} \beta_{n}
$$

Where $\beta_{\mathrm{H}}$ is the belief degree unassigned to any specific grade. If the value of $\beta_{\mathrm{H}}$ is zero then it can argued that there is an absence of ignorance or incompleteness. If the value of $\beta_{\mathrm{H}}$ is greater than zero then it can be inferred that there exists ignorance or incompleteness in the assessment.

\section{BRB IDS ARCHITECTURE}

Architectural design represents the structure of data and program components that are required to build a computer-based system. It also considers the pattern of the system organization, known as architectural style. BRB IDS adopts the three-layer architecture [15, 17], which consist of presentation layer, application layer and data processing layer as shown in Figure 1.

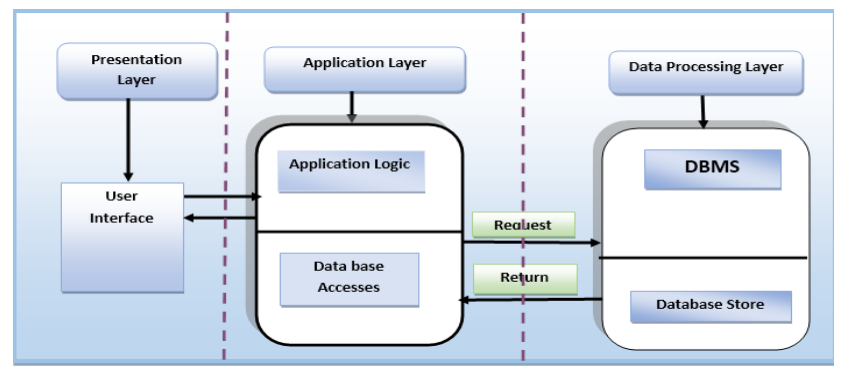

Figure 1. BRB IDS architecture

\subsection{System components}

The input clarification of input antecedent W11 (Security ward around),W12(Vandal Proof),W13( Open Location),W21(Expansion Capacity),W22(Parking Space),W23(Storey Number),W31(Neutral Location),W32(Traffic Access), W33(Public Transport Link), W41(Construction Cost),W42(Land Cost),W51(Land Risk),W52(Construction Risk),W53(Time Frame and delivery Speed) are transformed to referential value by equation (4), (5) on behalf of expert. The input clarifications of this BRB system transformed to referential is shown in Table 1.

Table 1. The input are transformed in referential value

\begin{tabular}{ccccccc}
\hline SI No. & Input antecedent & Expert belief & \multicolumn{4}{c}{ Referential value } \\
& & & Excellent & Good & Average & Bad \\
\hline 0 & W11 & 0.2 & 0.05 & 0.1 & 0.3 & 0.55 \\
1 & W12 & 1 & 1 & 0 & 0 & 0 \\
2 & W13 & 0.8 & 0.5 & 0.5 & 0 & 0 \\
3 & W21 & 0.5 & 0.1 & 0.8 & 0.1 & 0 \\
4 & W22 & 1 & 0.8 & 02 & 0 & 0 \\
5 & W23 & 0.9 & 0.86 & 0.14 & 0 & 0 \\
6 & W31 & 0.5 & 0.1 & 0.4 & 0.5 & 0 \\
7 & W32 & 1 & 0.8 & 0.2 & 0 & 0 \\
8 & W33 & 1 & 0.8 & 0.2 & 0 & 0 \\
9 & W41 & 0.4 & 0.1 & 0.5 & 0.4 & 0 \\
10 & $\mathrm{~W} 42$ & 0.5 & 0.5 & 0.4 & 0.1 & 0 \\
11 & W51 & 1 & 0.8 & 0.2 & 0 & 0 \\
12 & W52 & 0.6 & 0.5 & 0.3 & 0.1 & 0.1 \\
13 & W53 & 0.7 & 0.65 & 0.2 & 0.1 & 0.05 \\
\hline
\end{tabular}

\subsection{Knowledge base constructed using BRB}

In present paper, we worked on assessment process to select the suitable location for hospital establishment. In order to construct BRB knowledge base of this system we designed a BRB framework to site assessment according to domain expert. The BRB framework of suitable location assessment as illustrated in Figure 2, from the framework, it can be observed that input factors that determine suitable location for hospital. The BRB knowledge base has different traditional rule to assessment, which need to convert belief rules. 


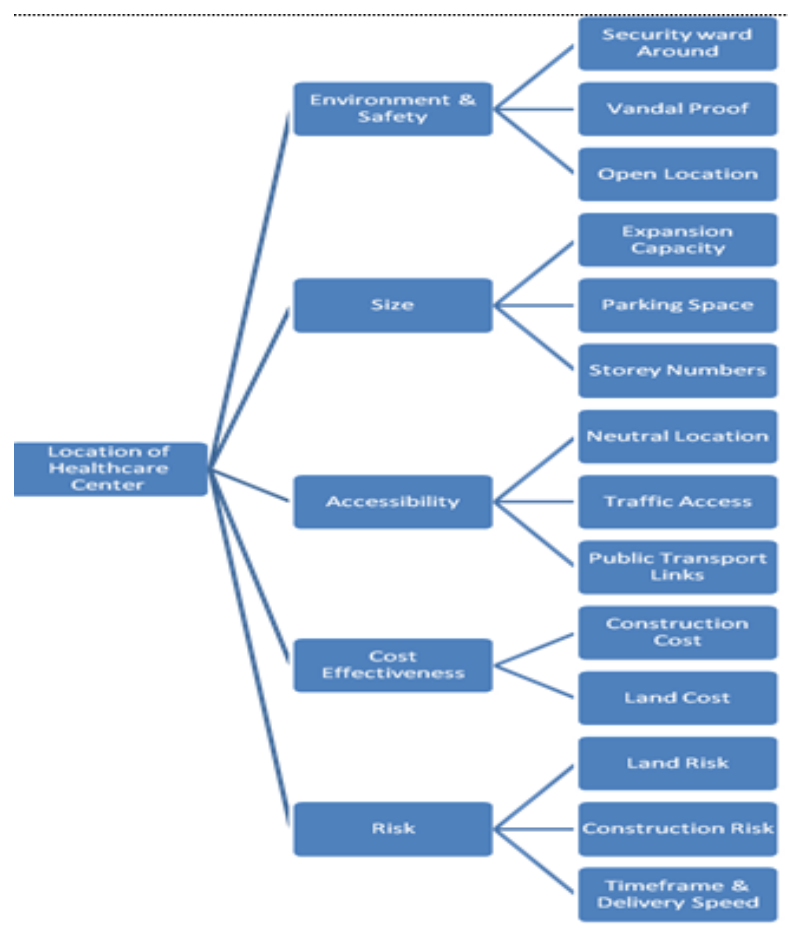

Figure 2. Hierarchical relationship among location evaluation variable

In such situations, belief rules may provide an alternative solution to accommodate different types and degrees of uncertainty in representing domain knowledge. A BRB can be established in the following four ways [16] (1) Extracting belief rules from expert knowledge (2) Extracting belief rules by examining historical data; (3) Using the previous rule bases if available, and (4) Random rules without any preknowledge. In this paper, we constructed initial BRB by the domain expert knowledge. This BRB consists of four sub-rule-bases namely environment and safety (W1), size (W2), accessibility (W3), cost effectiveness (W4), risk (W5) and location of healthcare center(S). W4 (Cost Effectiveness) sub-rule-base has three antecedent attributes. Each antecedent attribute consists of four referential values. Hence, this sub-rule-base consists of 16 rules. The entire BRB (which consists of six sub-rule bases) consists of $(64+64+64+16+64+1024)=1296$ belief rules. It is assumed that all belief rules have equal rule weight; all antecedent equal weight, and the initial belief degree assigned to each possible consequent by two expert from accumulated the data. To better handle uncertainties, each belief rule considered the three referential values are Excellent (E), Good (G), Average (A) and Bad (B).

Table 2: Initial belief rules of sub-rule-base (cost effectiveness)

\begin{tabular}{cccccccc}
\hline \multirow{2}{*}{ Rule No. } & Rule weight & \multicolumn{2}{c}{ IF } & \multicolumn{5}{c}{$\begin{array}{c}\text { THEN } \\
\text { Cost effetiveness } \\
\text { Good }\end{array}$} \\
\cline { 3 - 7 } & & W41 & W41 & Excellent & bad \\
\hline 0 & 1 & E & E & 1 & 0 & 0 & 0 \\
1 & 1 & E & G & 0.4 & 0.5 & 0.1 & 0 \\
2 & 1 & E & A & 0.5 & 0 & 0.5 & 0 \\
3 & 1 & E & B & 0.6 & 0.1 & 0.1 & 0.2 \\
4 & 1 & G & E & 0 & 0.8 & 0.3 & 0 \\
5 & 1 & G & G & 0 & 0.6 & 0 & 0 \\
6 & 1 & G & A & 0.33 & 0.66 & 0 & 0 \\
7 & 1 & G & B & 0 & 0.93 & 0.1 & 0 \\
8 & 1 & A & E & 0 & 0.8 & 0.2 & 0 \\
- & - & - & - & - & - & - & - \\
- & - & - & - & - & - & - & - \\
- & - & - & - & - & - & - & - \\
14 & 1 & B & A & 0.2 & 0 & 0.8 & 0 \\
15 & 1 & B & B & 0 & 0.06 & 0.93 & 0 \\
\hline An example of a belief rule taken from Table 2 illustrated in follow: &
\end{tabular}

作 2 illustrated in follow:

$\mathrm{R} 1$ : $I F$ W41 is ' $\mathrm{E}$ 'AND W42 is ' $\mathrm{E}$ '

THEN Cost Effectiveness (W2) is $\{\mathrm{E}(1.00), \mathrm{G}(0.00), \mathrm{A}(0.00), \mathrm{B}(0.00)\}$ 


\subsection{Inference engine using ER}

This BRB IDS designed using the ER approach $[15,17]$ which is described in section 2.2. It is similar to traditional forward chaining. The inference with a BRB using the ER approach also involves assigning values to attributes, evaluating conditions and checking to see if all of the conditions in a rule are satisfied. The BRB inference process using the ER approach described by the following steps are input transformation, calculation of the activation weight, calculating combined belief degrees to all consequents, belief degree update and aggregate multiple activated belief rules.

The inputs of data are of two types, objective and subjective. Input transformation of this system and input clarification are deduced in previous section and table 1 by using (4), (5). After the value assignment for antecedent, calculating the combined matching degrees between the inputs and the rule's antecedents, the next step is to calculate activation weight for each packet antecedent in the rule base using (6). The belief degrees in the possible consequent of the activated rules in the rule base are updated using (7). Then aggregating all activated rules using the ER approach to generate a combined belief degree in possible consequents using (9)(10). Then expected result of suitable location assessment was calculated from its different consequents of factors. Finally, presenting the system inference results of suitable location consequent which is not crisp/numerical value, then it is converted into crisp/numerical value for recommendation using (11).

\subsection{BRB IDS interface}

System interface is an intermediate position that represents the interaction between user and system. Figure 3 represents the BRB system interface of this paper.

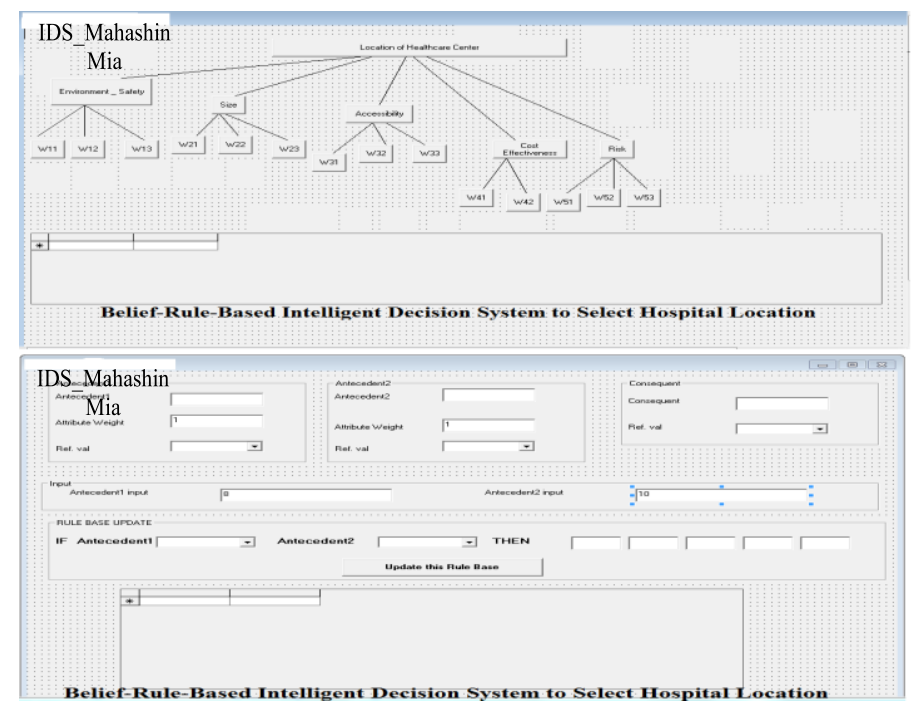

Figure 3. Graphical user interface of the IDS

\section{RESULTS AND DISCUSSION}

In the previous section, we have discussed about the RIMER method and how to implement it. Therefore, in this section we will look at the results from using this method on the different types of alternatives. Figure 4 shows the assessment distribution which must be done first by employing the transformation equation. Any measurements of quality can be translated to the same set of grades as the top attribute which make it easy for further analysis. The assessments given by the Decision Maker (DM) in Figure 5 are fed into IDS and the aggregated results are yielded at the main criteria level (Figure 5) 


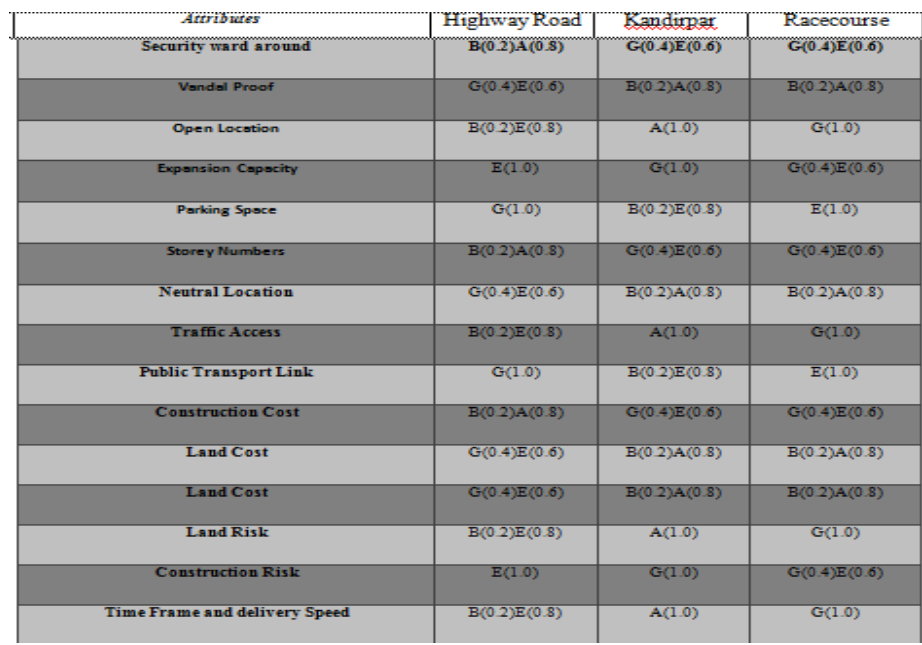

Figure 4. Assessment scores of suitable location based on sub criteria (E-excellent, G-good, A-average, B-bad)

\begin{tabular}{|c|c|c|c|c|c|}
\hline Alternative & Excellent & Good & Average & Bad & Total Dot: \\
\hline Highway Road & 0.80 & 0.10 & 0.10 & 0.00 & 1.00 \\
\hline Kandirpar & 0.15 & 0.45 & 0.20 & 0.20 & 1.00 \\
\hline Racecourse & 0.18 & 0.52 & 0.10 & 0.20 & 1.00 \\
\hline
\end{tabular}

Figure 5. The overall assessment (alternatives) (DoB-degree of belief)

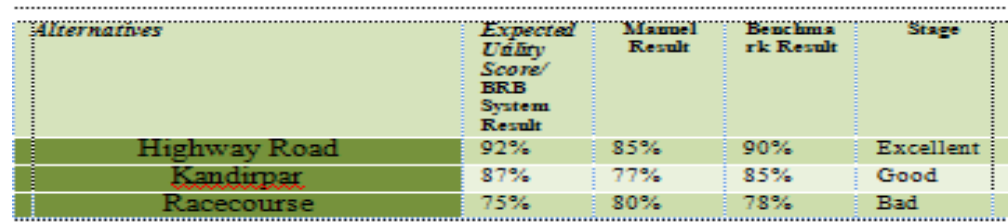

Figure 6. Overall assessment for suitable location

The three alternatives (location) simulated data set with assessment outcome is presented in Figure 6. This figure represents overall assessment outcome from location information. The result of this system is measured in percentage for recommendation. The output of this system was generated based on output utility (11). In this paper, the utility score of (100-90) \% assigned to 'Excellent', (85-89) \% assigned to 'Good', (80-84)\% assigned to 'Average' and (0-79)\% assigned to 'Bad'.

In the case study, the location assessment of three alternatives using this system, manual system and benchmark result is shown in Figure 6. The historical results were considered as benchmark. From Figure 4 it can be observed that IDS generated result has less deviation than from benchmark result. Hence, it can be argued that IDS output is more reliable than manual system. Therefore, it can be concluded that if the assessment of suitable location evaluation is carried out by using the IDS, eventually this will play an important role in taking decision to avoid uncertainty issue. The possible expected utilities of each alternative generated by the IDS (Figure 6) (based on the given utility values for each grade above). The alternatives ranked based on the expected utility. The ranking of alternatives is as follows: Highway Road > Kandipar > Racecourse

\section{CONCLUSION}

The development and application of a belief rule based IDS to choose suitable place by using attribute of different types of alternatives have been presented. The prototype IDS is embedded with a novel methodology known as RIMER, allows the handling of various types of uncertainty and hence, be considered as a robust tool can be utilized in selecting suitable location for hospital . Consequently, the prototype IDS 
can handle various types of uncertainties found in suitable area assessment domain knowledge as well as in attribute/criterion of a alternative. This system can also provide a percentage of recommendation, which is more reliable and informative than from the traditional expert's opinion. The prototype IDS can only is used to select good location by using attribute of a alternative.

\section{REFERENCES}

[1] M Sonmez, G. Graham and J. B. Yang and G D Holt, "Applying evidential reasoning to pre-qualifying construction contractors", Journal of Management in Engineering, vol.18, no.3, pp. 111-119, 2002.

[2] Lisa M., The Sage encyclopedia of qualitative research methods, Los Angeles, Calif.: Sage Publications, 2008.

[3] [Online]. http://www.pearson.ch/1449/9780273722595/An-Introduction-to-Geographical.aspx

[4] D. L. Xu and J. B. Yang, "Introduction to multi-criteria decision making and the evidential reasoning approach," Working Paper Series, No. 0106, Manchester School of Management, UMIST, pp. 1-21, 2001. [Online] Available: http://www.umist.ac.uk/management.

[5] L. Zadeh, "A simple view of the Dempster-Shafer theory of evidence and its implication for the rule of combination," The Al Magazine, vol. 7, no. 2, pp. 85-90, 1986.

[6] Kari Sentz and Scott Ferson, Combination of Evidence in Dempster-Shafer Theory, Sandia National Laboratories SAND 2002-0835, 2002

[7] Bragge, J, et al, "Bibliometric analysis of multiple criteria decision making/multiattribute utility theory," Multiple criteria decision making for sustainable energy and transportation systems, pp. 259-268, 2010.

[8] B. Yang and P. Sen, "Multiple attribute design evaluation of large engineering products using the evidential reasoning approach," Journal of Engineering Design, vol. 8, no. 3, pp. 211-230, 1997

[9] Mahmud, T , Hossain, M.S, “An Evidential Reasoning-based Decision Support System to Support House Hunting," International Journal of Computer Applications, vol. 57, no. 21, pp. 51-58, 2012.

[10] Yang, J. B., Liu, J., Wang, J., Sii, H. S. \& Wang, H. W., "Belief rule-base inference methodology using the evidential reasoning approach -RIMER," IEEE Transactions on Systems Man and Cybernetics Part A-Systems and Humans, vol. 36, no. 2, pp. 266-285, 2006.

[11] D.L. Xu, et al, "Inference and learning methodology of belief-rule-based expert system for pipeline leak detection," Expert Systems with Applications, vol. 32, no. 7, pp. 103-113, 2007

[12] J. Yang, et al, "Optimization models for training belief-rule-based systems," IEEE Transactions on Systems, Man, and Cybernetics - Part A: Systems and Humans, vol. 37, no. 4, pp. 569-585, 2007

[13] Patwary, M.J.A , Akter, S, Mahmud, T., "An expert system to detect uterine cancer under uncertainty," IOSR Journal of Computer Engineering (IOSR-JCE), vol. 16, no. 5, pp. 36-47, 2014

[14] A. M. Nonvich and T. B. Turksen, "A model for the measurement of membership and the consequences of its empirical implementa-tion," Fuzzy sets and systems, vol. 12, no. 1, pp. 1-25. 1984.

[15] Roger S. Pressman, Software engineering: a practitioner's approach, 6th ed. p. cm.(McGraw-Hill series in computer science) Includes index, pp. 373- 374, 2005

[16] Mahmud,T.;Rahman,K.N.;Hossain,M.S., "Evaluation of job offers using the evidential reasoning approach," Global Journal of Computer Science and Technology, Vol. 13, no. 2, 2013.

[17] Mahmud,T ,Mia ,Mahashin, "Intelligent decision system for evaluation of job offers," 1st National Conference on Intelligent Computing and Information Technology( NCICIT ), 2013

\section{BIOGRAPHIES OF AUTHORS}

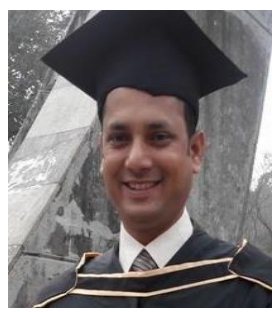

Mr. Md. Mahashin Mia received his Bachelor of Science (B.Sc) and Master of Engineering (M.Engg.) degree from the Department of Computer Science and Engineering at University of Chittagong, Chittagong, Bangladesh. In profession, he is an assistant registrar at Chittagong Veterinary and Animal Sciences University, Chittagong, Bangladesh. He is now conducting his M.Phil research work at Department of Computer Science and Engineering in University of Chittagong, Chittagong, Bangladesh. His current research interest lies in the field of machine learning arena. 

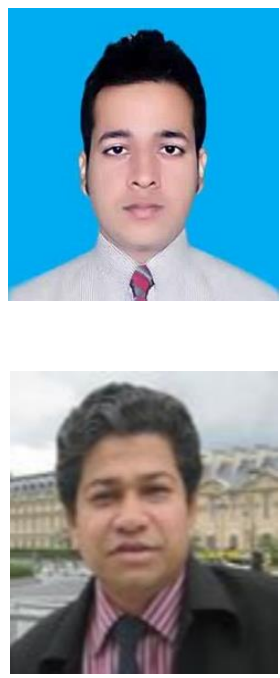

Mr. Atiqur Rahman received his Bachelor of Science (B.Sc) and Master of Engineering (M.Engg.) degree from the Department of Computer Science and Engineering at University of Chittagong, Chittagong, Bangladesh. In profession, he worked in the Department of Computer Science and Engineering, University of Chittagong, Bangladesh as an Assistant Professor since April 2016.Formar he was a lecturer in the Department of Computer Science and Engineering, University of Chittagong, Bangladesh. He is now conducting his Ph.D. research works under the Chinese Government Scholarships (CGS) Program at Chongqing University of Posts and Telecommunications, Chongqing, China. His current research interest lies in the field of edge computing based IoT systems.

Dr. Mohammad Shahadat Hossain is a Professor of Computer Science and Engineering of Chittagong University, Bangladesh. He earned his M.Phil and Ph.D. from the University of Manchester Institute of Science and Technology (UMIST). He has published several scholarly articles in learned refereed journals. He awarded prestigious Commonwealth Academic Staff Fellowship and European Commission sponsored Erasmus Mundus Fellowship in 2009 and 2011 respectively. He successfully completed a number of research projects as a co-investigator. His current research area includes the modeling of risk and uncertainty using evolutionary computing techniques. Investigation of pragmatic software development tools and methods for information system in general and for GIS in particular are also his area of research. He is continuing his research at the intersection of computing and real world issues like economics, business, engineering and environment. He is the innovator of SDA (Spatial Domain Analysis) approach used to facilitate socio-economic research. In addition, he earned reputation as a Tawhidi scientist, who uses this method to develop pragmatic computer model of reality. His jointly authored book entitled "Computing Reality", published by Aoishima Research Institute (blue ocean press) in Tokyo, Japan, contributed significantly to enrich the knowledge of computer science. 